\title{
Payer type and the returns to bypass surgery: evidence from hospital entry behavior
}

\author{
Michael Chernew ${ }^{\mathrm{a}, *}$, Gautam Gowrisankaran $^{\mathrm{b}}$, A. Mark Fendrick ${ }^{\mathrm{c}}$ \\ a Department of Health Management and Policy, University of Michigan and NBER, MI, USA \\ ${ }^{\mathrm{b}}$ Department of Economics, University of Minnesota, (MN) Federal Reserve Bank of San Francisco, \\ (CA) and NBER, USA \\ c Department of Internal Medicine, Consortium for Health Outcomes Innovation and Cost Effectiveness Studies, \\ University of Michigan, MI, USA
}

Received 31 January 2001; received in revised form 01 December 2001; accepted 12 December 2001

\begin{abstract}
In this paper, we estimate the returns associated with the provision of coronary artery bypass graft (CABG) surgery, by payer type (Medicare, HMO, etc.). Because reliable measures of prices and treatment costs are often unobserved, we seek to infer returns from hospital entry behavior. We estimate a model of patient flows for CABG patients that provides inputs for an entry model. We find that FFS provides a high return throughout the study period. Medicare, which had been generous in the early 1980s, now provides a return that is close to zero. Medicaid appears to reimburse less than average variable costs. HMOs essentially pay at average variable costs, though the return varies inversely with competition. (C) 2002 Elsevier Science B.V. All rights reserved.
\end{abstract}

Keywords: Entry models; Hospital margins; Managed care

\section{Introduction}

In this paper, we seek to estimate the returns associated with the provision of coronary artery bypass graft (CABG) surgery by payer type. CABG surgery is used to treat coronary artery disease, which is a leading cause of death, morbidity and expense in the United States. $\mathrm{CABG}$ is an expensive, technologically intensive procedure provided to over 350 thousand Americans annually, with utilization growing at over $9 \%$ a year. ${ }^{1}$ Returns, which capture

\footnotetext{
* Corresponding author. Present address: University of Michigan, School of Public Health, 109 Observatory, Ann Arbor, MI 48109-2029, USA. Tel.: +1-734-936-1193; fax: +1-734-764-4338.

E-mail addresses: mchernew@umich.edu (M. Chernew), gautam@econ.umn.edu (G. Gowrisankaran), amfen@umich.edu (A.M. Fendrick).

1 National Center for Health Statistics (1997) and National Center for Health Statistics (1986).
} 
all the benefits to the hospital associated with the per-case provision of CABG, will reflect financial and other rewards for use of this procedure.

Variations in returns raise both efficiency and equity concerns. Overly generous payments by prominent insurers such as Medicare may induce excessive entry by hospitals into the market for $\mathrm{CABG}$ as well as over-usage among hospitals that provide CABG. The possibility of excessive provision of CABG is of particular importance since the rapid rise in health care expenditures in recent years has been attributed to the growing diffusion and utilization of expensive high technology services such as CABG (Cutler and McClellan (1996)).

Variations in payment rates among insurers, which would generate variations in returns, have led to charges that some payers are not paying their 'fair share'. Considerable attention has been devoted to measuring 'cost shifting', the practice of raising the prices to some payers to cover insufficient payments from other payers (Hadley and Feder (1985), Morrisey (1995)). If the Medicare payment rates are too high, taxpayers are subsidizing health care providers, or perhaps subsidizing individuals covered by insurers who pay less for care. Large, self-insured employers, who often pay more for services than managed care plans, complain that they are subsidizing other employers with different insurance types. To the extent that cost savings associated with HMOs reflect price advantages made possible by generous payments from other payers, HMO induced savings may be short-lived.

The direct way to uncover margins, a prime component of returns, is to find or estimate prices and costs. While Medicare payment rates for CABG are publicly available, ${ }^{2}$ prices paid by other insurers, particularly HMOs, are difficult to observe. It is generally thought that commercial and Blue Cross insurers pay the most for services, followed by Medicare, managed care plans, and Medicaid.

Even though we have a general sense of relative payment generosity, observation of costs would be required to determine whether some payers, such as managed care plans, are paying below costs or merely covering the expenses of their enrollees. Relative margins may not map directly to payment rates because of variation in costs. Measurement of costs by payer and service type is inherently difficult because hospital accounting systems and case-mix differences make it difficult to directly attribute costs to specific patients or services. Costs for different types of patients are likely to be very different.

While it is difficult, if not impossible, to estimate returns directly, there is an alternative method to infer returns, which is to observe entry into the market for CABG surgery. We make the assumption that hospitals that do not already provide $\mathrm{CABG}$ will choose to enter into the $\mathrm{CABG}$ market only if they receive sufficient returns. Returns are then linked to entry behavior: the higher the variable returns are, the more likely a hospital will be to enter into the $\mathrm{CABG}$ market. Over the period of our study the number of CABGs rose about $5 \%$ per year and the number of hospitals offering CABG rose from 84 to 126 . About one-third of potential entrants for CABG entered over the sample period. This provides us with a reasonably sized data set on entry behavior.

Our use of entry as a tool to uncover underlying industry parameters has support in the empirical industrial organization literature (Bresnahan and Reiss (1991), Berry (1992)). In contrast to these earlier works, we construct a model where we endogenously determine and test for the appropriate market definition. We are able to do this by combining detailed

\footnotetext{
${ }^{2}$ In 1991, average Medicare reimbursement for CABG was US\$ 32,117 (Cutler and McClellan (1996)).
} 
patient discharge data with the hospital entry data. Additionally, as we discuss below, our results appear to be confirmed by economic theories and independent accounting data, which validates the use of entry as a tool to uncover fundamental firm parameters. Thus, one can think of using entry to examine many aspects of hospital behavior where prices or costs are not estimable, such as economies of scope between different services and the nature of competition between insurance providers and hospitals.

The focus of this research is on analyzing the variable returns to CABG surgery. Our entry model is based on a hospital 'return' function that assumes that average returns for CABG are different for each of six payer types (Medicare, Medicaid, HMO, FFS, other public coverage and other private coverage). ${ }^{3}$ We express total variable returns as the sum of the returns received from patients of each payer type. These returns are in turn given by the volume of patients by payer type, times the average return for that payer type. As we do not observe volumes for hospitals that do not enter, we need to predict volumes. From our return function, if a payer is paying a positive average return, this will generate a positive correlation between volume for that payer type and the probability of entry, and vice versa. Thus, assuming that we can approximate predicted volumes, we can infer relative average returns by regressing the volumes by payer type on the entry decision, using a duration model approach.

We predict volumes for potential entrants using conditional logit models of patient flows for $\mathrm{CABG}$ services. We allow hospital choice for $\mathrm{CABG}$ to be influenced by the insurance type of the patient, distance from the patient to the hospital, and various hospital attributes such as ownership, number of beds, and lagged cardiac and non-cardiac volume. Using our estimated logit coefficients, we then predict volumes for potential entrants by simulating the addition of the entrant into the market and calculating expected patient flows to that hospital. Econometric tests confirm that we are able to closely estimate volume, even for entrants.

The identification of returns in our model is based on the differences in expected patient flows by payer type across hospitals (HMOs, FFS, etc.). For example, if entry is likely for hospitals that would receive many FFS patients conditional on entry, we will tend to infer that FFS coverage is associated with positive returns. The differences in expected patient flows by payer types are in turn identified by the level of competition for CABG patients from nearby hospitals, the number of patients by payer type in the geographic area surrounding a hospital, and the probability of admission by payer type for non-cardiac services and cardiac services in previous years.

Identification from the geographic distribution of patients is common in this literature (Cutler and McClellan, 1996; Baker and Wheeler, 1998; Spetz and Baker, 1999). Our model builds on this identification by adding in the effect of competition and specialization by payer type. Thus, we will view two hospitals in areas that have the same number of FFS patients differently if there are different numbers of existing or potential CABG providers in those areas, or if those hospitals admit different numbers of FFS patients for non-cardiac services.

\footnotetext{
3 The return function serves as a profit function, but conceptually allows non-financial benefits to be associated with provision of CABG and is perhaps more semantically appropriate given the prevalence of not-for-profit hospitals in our sample.
} 
Our results confirm the general opinion that average returns from FFS insurers are the greatest,${ }^{4}$ followed by Medicare, HMOs, and Medicaid. Consistent with published ProPAC figures, ${ }^{5}$ our estimates suggest that at the start of our study period Medicare provided a large return that had disappeared by the end of the study period. Economic theory suggests that those payers willing to shop for care would receive prices approximating costs and our results confirm this intuition, as HMOs appear to be providing hospitals with returns close to zero. Finally, unlike returns for other payers, returns from HMOs vary inversely with the degree of market competitiveness, consistent with competitive bidding for hospital services by HMOs.

We explore several econometric issues, including the specification of the duration model, the ability of entry to expand the market size, the possibility of unobserved market traits that are correlated with both entry and payer mix, and the potential endogeneity of predicted volume. Estimates accounting for these factors support the conclusions reached in our primary analysis.

The remainder of this paper is divided as follows. In Section 2, we detail the model. Section 3 outlines our data and construction of entry variables. Section 4 provides our results. Section 5 concludes.

\section{Model}

In order to estimate CABG returns, we specify two different models, a patient flow model and a hospital entry model. For the entry model, we take as our sample the set of potential entrants into the market for CABG services and regress the decision of whether to enter on anticipated volume by payer type, controlling for hospital-specific attributes. We define potential entrants to be the set of hospitals that were admitting patients in the current year, that did not perform CABGs in the previous year, and that provided some cardiac service (cardiac catheterization, angioplasty or CABG) at some point in the period of our study. ${ }^{6}$ We estimate the entry model using 9 years of data. Our primary interest is to estimate the coefficients of the entry model. With the assumptions of optimizing behavior and rational expectations, these coefficients are interpretable as average variable returns.

In order to estimate the coefficients of the entry model, we need measures of expected volume by payer type for the set of potential entrants. This is where we use the patient flow model. We estimate choice of hospital using a reduced-form conditional logit framework where choice is assumed to be a function of the patient's insurance type, distance to each hospital and various hospital characteristics. To calculate volume for potential entrants, we then make use of the fact that potential entrants are all existing hospitals, and hence have well-defined values for the variables that we assume affect hospital choice, and thus patient

\footnotetext{
${ }^{4}$ We use the term FFS to refer to Commercial and Blue Cross/Blue Shield plans. This category includes a variety of partly managed care plans such as Point of Service plans, which may not reimburse on a fee-for-service basis.

5 Prospective Payment Assessment Commission (1995).

6 Virtually all potential entrants provided cardiac catheterization, a less technologically advanced cardiac service, prior to entering the market for CABG. Thus our set of potential entrants is largely the set of hospitals providing catheterization but not CABG. We also experimented by adding all hospitals with 200 or more beds to the list of potential entrants. This did not substantively change any results.
} 
flows. Using our estimated discrete choice model, we simulate the addition of a potential entrant into the industry, and calculate its predicted volume by payer type conditional on it entering. By doing this for every potential entrant separately, we then obtain the expected volume for each potential entrant which decides to enter. These we then use as explanatory variables for our entry model.

We now proceed to detail the entry model and patient flow model.

\subsection{A model of entry}

We posit an optimizing model of entry. For the purposes of this study, we will assume static optimization, so that returns in the year subsequent to entry do not affect the entry decision. This is equivalent to the assumption that hospitals have a discount factor of 0 . While this assumption is also likely to be false, hospitals have the option to enter in the subsequent period. If the decision to enter does not cause the state space to change, then firms should only examine the increment to returns from current period CABG volume in their entry decision. The only caveat to this is that the decision to not enter may precipitate a rival to enter, which would then change the state space by lowering the future returns from entry.

We write total returns for a potential entrant $j$ in year $t$ as

$$
\mathrm{TR}_{j, t}=\sum_{h=1}^{m} P_{h, j, t} Q_{h, j, t}-C\left(Q_{1, j, t}, \ldots, Q_{m, j, t}\right)+K_{j, t},
$$

where $h$ indexes $m$ payer types, which are Medicare, Medicaid, HMO, FFS, other public and other private; $P_{h, j, t}$ denotes average per CABG reward (largely price) from payer $h$ to hospital $j$ in year $t ; Q_{h, j, t}$ denotes average quantity from patients with insurance from payer $h$ to hospital $j$ in year $t ; C()$ denotes variable costs; and $K_{j, t}$ denotes the sum of fixed and sunk benefits of entry for hospital $j$ in year $t$.

We assume that variable costs are separable by payer type. Thus $C\left(Q_{\left.1, j, t, \ldots, Q_{m, j, t}\right)}\right.$ becomes $\sum\left(\mathrm{AVC}_{h, j, t} Q_{h, j, t}\right)$, where AVC denotes average variable costs. With this assumption, the returns equation reduces to:

$$
\mathrm{TR}_{j, t}=\sum_{h=1}^{m} Q_{h, j, t}\left(P_{h, j, t}-\mathrm{AVC}_{h, j, t}\right)+K_{j, t} \equiv \sum_{h=1}^{m} Q_{h, j, t} R_{h, j, t}+K_{j, t},
$$

where $R_{h, j, t}$ is defined to be the average return by payer type and hospital.

We assume that hospitals will enter if total variable returns exceed the threshold $-K_{j, t}$. Profit-maximization is consistent with $K_{j, t}$ equal to the negative of the fixed and sunk costs of entry. However, many hospitals are not-for-profit and these hospitals may have different thresholds for entry. Thus, we allow $K_{j, t}$ to be a function of hospital characteristics that might influence the objective function including ownership status (e.g. not-for-profit) and medical school affiliation.

Specifically, we define:

$$
K_{j, t}=Z_{j, t} \gamma+e_{j, t},
$$

where $Z_{j, t}$ includes hospital-specific variables that might influence the objective function as well as year dummies, and $e_{j, t}$ represents the unobservable component of $K_{j, t}$. The year 
dummies will capture trends in entry costs as well as changes in the fixed and sunk benefit of entry for the average potential entrant caused by changes in the pool of potential entrants over time.

We make one major simplifying assumption in our base model that we later relax: that the degree of market power is similar enough within a payer type so that the average returns are the same within payer type across different hospitals and different years. For Medicare (where prices are fixed), this implies that hospital costs for Medicare patients do not vary by hospital; for other payer types the assumption is similar. Given our assumptions, hospital $j$ will enter at the first year $t$ when:

$$
\sum_{h=1}^{m} Q_{h, j, t} R_{h}+Z_{j, t} \gamma+e_{j, t}>0
$$

From (4), one can see that in order to estimate average returns, it is necessary to be able to predict the quantities that potential entrants face should they choose to enter. We do not use a model of hospital pricing behavior to estimate $Q_{h, j, t}$. Instead, our approach is to estimate $Q_{h, j, t}$ using a reduced-form patient flow model that we detail in Section 2.2.

The variance of $e_{j, t}$ is not identified from the data. Hence, for our base results, we normalize $e_{j, t} \sim N(0,1)$, i.i.d. In this case, the estimated returns coefficients should be interpreted as their true values divided by a common constant, the standard deviation of $e_{j, t}$. With this distributional assumption, we can write the conditional probability of entry for a potential entrant as a standard probit:

$$
\operatorname{Pr}\left(\text { entry }_{j, t} \mid j \text { not in at } t-1\right)=\Phi\left(\sum_{h=1}^{m} Q_{h, j, t} R_{h}+Z_{j, t} \gamma\right),
$$

where ' $\Phi$ ' indicates the standard normal cdf. We estimate this specification by using maximum likelihood on the set of potential entrants. Since potential entrants are dropped from the sample once they enter, (5) is a duration model with a time-varying hazard, where the 'hazard' is entry into CABG.

To verify the robustness of our results, we also examine a couple of alternate specifications similar to (5). First, we estimate a version where we relax the i.i.d. assumption on $e_{j, t}$. Specifically, we allow the error terms for a given hospital across time to follow an AR(1) process, which implies that

$$
\operatorname{Corr}\left[e_{j, t}, e_{j, t-s}\right]=\rho^{|t-s|},
$$

where $\rho$ is an additional parameter to estimate. This model will capture unobserved hospitalspecific effects. Because we have nine time periods, a direct maximum likelihood estimation of the serially correlated probit model would involve nine-dimensional normal integration for each hospital, which is not computationally feasible. Instead, we use the method of simulated likelihood combined with the GHK algorithm (Geweke (1989), Hajivassiliou et al. (1996), and Keane (1994)) to efficiently simulate the normal densities. 
Second, we estimate a Cox proportional hazard model. In this model, the hazard of entry for hospital $j$ at time $\hat{t}$ that lies between $t-1$ and $t$ is given by

$$
f\left(\operatorname{entry}_{j, \hat{t}} \mid j \text { not in at } \hat{t}\right)=h_{0}(\hat{t}) \exp \left(\sum_{h=1}^{m} Q_{h, j, t} R_{h}+Z_{j, t} \gamma\right),
$$

where $h_{0}(\hat{t})$ is a non-parametric function of time. The hazards stemming from (5)-(7) are similar, particularly since the probit specifications contain time dummies. D' Agostino et al. (1990) and Allison (1995), demonstrate the close correspondence between these two types of duration modeling specifications. We estimate (7) because it provides a different functional form, and one that is more standard, for the hazard. However, unlike the probit specifications, we are not aware of any functional form for the $e_{j, t}$ 's in (4) that would generate (7). The Cox model can be estimated via conditional maximum likelihood.

We limit our sample to those hospitals that had not entered by the start of our study period, 1986. This excludes 85 hospitals, or two-thirds of hospitals that ultimately entered. We do not address this left censoring for both conceptual and practical reasons. Conceptually, the motivation for early entrants may differ from that of late entrants. Hospitals strongly inclined to adopt new technology regardless of returns are likely to be early adopters. Therefore, we think that the coefficients may not be stable between early and late entrants. On a practical level, our model posits entry as a function of time-varying regressors, including estimated volumes. Although we can observe the year of entry for all hospitals from AHA data (even those that entered before our study period), we do not have the data necessary to estimate predicted volume prior to our study period.

We estimate (5)-(7) using predicted values for $Q_{h, j, t}$ for all potential entrants, where these predicted volumes are derived from our patient flow model, as described in Section 2.2. We use predicted volume instead of actual volume conditional on entry because we do not observe the volume that non-entrants would have received had they entered. Moreover, actual volume in the year of entry is a poor measure of the steady state volume, since hospitals often enter in the middle of a year.

Our predicted values for $Q_{h, j, t}$ are a proxy for the true expected annual value of $Q_{h, j, t}$. The difference between the predicted and true volumes is either proxy error or measurement error, depending on whether the difference is correlated with the true or predicted volume, respectively. Measurement error would occur if our true volumes were imprecisely estimated due to a limited sample size. Because our predicted volumes are based on our patient flow model with over 20,000 patients in each year, we feel that measurement error will be negligible. Thus, any difference is likely attributable mostly to unobserved hospital traits that affect true expected volume but are uncorrelated with our predicted volume, i.e. proxy error. Unlike measurement error, proxy error will not bias the coefficients but will simply be added to the entry model error.

There are also potential concerns that the predicted volumes may be biased in a variety of ways. For instance, there is the possibility that actual entrants may have some unobserved advantage that leads them to receive a higher volume than potential entrants who do not enter. Additionally, the predicted volumes may be endogenous because of competitive interactions. In Section 4, we try to assess the magnitude of these issues and correct for them. 
Lastly, we note that we have made a number of simplifying assumptions for our base case model in (5), which we later relax. First, we assume that average returns for a given payer type do not vary across time and hospital. Because the level of competition may differ for different hospitals, returns may vary by hospital for some payer types. Additionally, exogenous changes in institutional structures (e.g. Medicare payment rate changes) may result in a variation in average returns across time. To mitigate this problem we examine specifications where returns are allowed to be a function of time or of the level of hospital competition.

\subsection{Predicting volume}

Our estimates of predicted volume $Q_{h, j, t}$ are based on a utility maximizing model of hospital selection. The underlying econometric model is based on the assumption that the utility associated with any particular hospital for CABG reflects hospital characteristics, the distance from the patient's residence to the hospital, and the patient's insurance type. The utility function is a composite of patient, physician, and insurer utility, all of which influence hospital choice. The setup is analogous to the conditional choice model of McFadden (1973, 1974). We specify the utility derived if individual ' $i$ ' chooses hospital ' $j$ ' as

$$
U_{i j}=X_{I_{i}, j} \beta_{I_{i}, T_{i}}+f\left(D_{i j}\right) \lambda_{I_{i}, T_{i}}+\varepsilon_{i j},
$$

where $I_{i}$ is patient $i$ 's insurance type (which is one of six listed above), $T_{i}$ is the year in which the patient receives treatment (ranging from 1986 to 1994), $X_{I_{i}, j}$ is a vector of hospital characteristics that can vary by payer type (which is detailed in Section 3 ), $D_{i j}$ is the distance in kilometers from patient $i$ 's residence to hospital $j,(\beta, \lambda)$ are vectors of parameters, and $\varepsilon_{i j}$ is a Type I extreme value stochastic term capturing preferences for unobserved hospital attributes.

Explanatory variables are intended to capture hospital attributes that might attract patients, their physicians, or even their insurers. Perhaps most important of these variables is the distance between the patient's residence and the hospital, $D_{i j}$. This distance is measured as the straight-line distance between the zip code centroid of the patient's residence and the hospital. Other studies have found distance to be a primary determinant of patient flows (Luft et al. (1990), Chernew et al. (1998), Burns and Wholey (1992)). Our model includes two measures of distance; specifically, we let $f\left(D_{i j}\right)=\left(\log \left(1+D_{i j}\right),\left[\log \left(1+D_{i j}\right)\right]^{2}\right)$.

If individual $i$ receives treatment at hospital $j$, it is assumed that the utility associated with hospital $j$ must exceed that of all other hospitals that person $i$ could have chosen. In our primary analysis, we do not allow for an outside alternative for the practical reason that we only observe the set of patients who are treated for CABG. In our sensitivity analysis, we adjust for the percentage of heart disease patients receiving CABG.

We define $Y_{i j}=1$ when individual $i$ is treated at hospital $j$ and $Y_{i j}=0$, otherwise. Then, we obtain the usual logit formula:

$$
\operatorname{Pr}\left\{Y_{i j}=1\right\}=\frac{\exp \left(X_{I_{i}, j} \beta_{I_{i}, T_{i}}+f\left(D_{i j}\right) \lambda_{I_{i}, T_{i}}\right)}{\sum_{k \in C_{i}} \exp \left(X_{I_{i}, k} \beta_{I_{i}, T_{i}}+f\left(D_{i k}\right) \lambda_{I_{i}, T_{i}}\right)},
$$

where $C_{i}$ is the choice set for patient $i$. 
The choice set $C_{i}$ for individual $i$ is comprised of all hospitals offering CABG within a $120 \mathrm{~km}$ market area of her residence. This distance threshold captures over $90 \%$ of all patients. Many of the remaining patients likely have miscoded distances or received care as part of an emergency episode when they were away from home.

We estimate (9) using maximum likelihood. Given the logit error term, we can estimate (9) separately by payer type and year. Estimated coefficients from (9) can be used to predict volume by payer for potential entrants. We assume rational expectations: potential entrants know which other hospitals will be in the market and the aggregate volume of CABG. Predicted volume for hospital $j$, if it enters, is computed as:

$$
\hat{Q}_{h, j, t}=\sum_{i \in M_{h, j, t}} \frac{\exp \left(X_{h, j} \hat{\beta}_{h, t}+f\left(D_{i j}\right) \hat{\lambda}_{h, t}\right)}{\sum_{k \in C_{i} \cup j} \exp \left(X_{h, k} \hat{\beta}_{h, t}+f\left(D_{i k}\right) \hat{\lambda}_{h, t}\right)},
$$

where $M_{h, j, t}$ is the set of all patients residing within a $120 \mathrm{~km}$ market area of hospital $j$ at time $t$ who are of insurance type $h$.

There are a couple of features about our modeling of hospital choice that are of potential concern. First, the logit distribution of the $\varepsilon$ 's in (8) implies independence of irrelevant alternatives (IIA). IIA implies that the probability of choosing a given hospital relative to another hospital is independent of the other hospitals in the patient's choice set. If some hospitals are closer substitutes to a given hospital than others, IIA will be violated. Although IIA will apply at the individual level, this fear is somewhat mitigated by the fact that we include geographical location and insurance type. Because of this, IIA will not hold at the aggregate level. Recall from (5) that we are only interested in aggregate volume by payer type for each hospital. Moreover, Phibbs et al. (1993) estimated similar models of patient flows for birthing services and could not reject the hypothesis of IIA.

Another issue is the possibility of unobserved hospital-specific attributes uncorrelated with the $X$ 's. Such hospital-specific factors will generate correlations among the $\varepsilon$ 's. This will result in overestimated precision on the coefficients from the patient flow model. Alternative methods, using grouped data, could be used to address this problem (Berry (1994)), but studies of patient flows suggest that estimation using individual level data is more stable (Garnick et al. (1989)). Moreover, our inferences from the entry model do not require use of the variance of the coefficients of the patient flow model. Therefore, underestimated standard errors from the patient flow model do not affect our conclusions. To check the stability of our estimates, we examine the correlations between predicted volumes generated from coefficients estimated on data from different years. For example, we measure the correlation between predicted 1994 volume using coefficients based on 1994 data with predicted 1994 volume using coefficients based on 1992 data.

Lastly, we note that we did not include measures of prices in our choice equation. This decision was made for two reasons. First, prices are not observed for most payer types and hence cannot easily be used. ${ }^{7}$ Second, we view the hospital choice equation as a reduced-form equation that encapsulates optimizing pricing behavior. As an endogenous variable chosen by hospitals, price should not be included in the set of explanatory variables.

\footnotetext{
${ }^{7}$ Medicare prices are observed. Because Medicare does not steer patients to hospitals, these prices are unlikely to affect patient flows.
} 
Despite these concerns about the entry model, our inference regarding returns requires primarily that we approximate predicted patient volumes well. A more detailed model might explain patient flows better, but as the results presented below indicate, our existing model does a reasonable job and is sufficient for our purposes.

\section{Data}

Our data come primarily from hospital discharge records for the state of California supplied by the Office of Statewide Health Planning and Development. The data contain information on every discharge from hospitals in California between 1984 and 1994 (about 300,000). For each discharge, we observe a hospital ID, principal source of payment (payer type), five procedure codes, and the zip code of the patient's residence. We merged this with hospital-specific data from the American Hospital Association to capture the zip code of the hospital and hospital characteristics. We used the Census TIGER database and standard formulas to compute straight-line distances between hospitals and the zip code centroid of patient residences.

Patients were identified as $\mathrm{CABG}$, percutaneous transluminal coronary angioplasty (PTCA) or cardiac catheterization patients if any of the International Classification of Diseases Ninth Revision (ICD-9) procedure codes indicated one of these procedures. ${ }^{8} \mathrm{We}$ were broad in our definition of procedure codes because we want to capture all of the possible returns from procedures potentially performed using the CABG facilities. Some of the included procedures could be performed even without the specialized facilities that we are studying.

We discarded patients residing outside of California or with missing distance data. We also omitted patients who traveled farther than $120 \mathrm{~km}$ for care. Lastly, we omitted patients who were treated at hospitals that were not 'in' the CABG market. Collectively these sample restrictions reduced our sample by $10 \%$.

In order to estimate our model of entry, we need to measure which hospitals were active in the market in any year. We infer participation in the CABG market by aggregating the discharge data to the hospital level, and observing the number of patients receiving $\mathrm{CABG}$ at each hospital.

We considered hospitals to be 'in' the market for CABG in any particular year if they performed more than $20 \mathrm{CABG}$ in that year. We used the 20 procedure threshold because our broad definition of CABG and miscoding may erroneously indicate entry and very low volumes may be performed at facilities not genuinely 'in' the market for providing CABG. This threshold minimized the problem of erratic entry behavior; hospital entering, exiting, and re-entering the market over our study period. ${ }^{9}$

\footnotetext{
${ }^{8}$ For CABG, we use ICD-9 procedure codes of 35.1x-35.7x, 35.90-35.95, 35.98, 35.99, 36.03, 36.1x, 36.2x, 36.9x, 37.1x, 37.24, and 37.3x. For PTCA, we use codes of 35.96, 36.01, 36.02, 36.05 and 36.06. For cardiac catheterizations, we use codes of $36.04,37.21-37.23,37.25,88.50,88.52-88.57$.

${ }^{9}$ Despite use of the 20 procedure threshold, there were three hospitals that displayed erratic entry behavior. Two of these three hospitals performed fewer than 20 procedures for a year or two after having higher volume earlier and then returned to high volume. We considered those hospitals to be 'in' the market from the first year at which they performed 20 or more procedures. The third hospital was a Kaiser facility whose annual CABG volume fluctuated between 3 and 20. We considered it not to be 'in' the CABG market during our study period.
} 
The final data issue we addressed was treatment of hospitals owned by the Kaiser Foundation Health Plan. Kaiser is by far the dominant HMO in California and owns and operates its own hospitals. These facilities largely treat Kaiser enrollees. For some tertiary services, including $\mathrm{CABG}$, Kaiser supplements its capacity by contracting with other hospitals to provide the services to its enrollees. We cannot identify Kaiser enrollees, but we can identify Kaiser owned facilities, most of which do not offer CABG. Entry by Kaiser Foundation Hospitals may reflect different factors than entry by other hospitals because entry decisions are made centrally and because Kaiser is primarily concerned with its enrollees. When Kaiser hospitals are excluded from the base model, estimated returns from HMO payers switch sign (from negative to positive), but remain statistically insignificant. Our other conclusions are robust to exclusion of these Kaiser hospitals from the entry model. Because of space limitations, we do not present the results that exclude Kaiser hospitals any further. ${ }^{10}$

Explanatory variables in our patient flow model $\left(X^{\prime}\right.$ s) include several variables measuring ownership status, teaching status, and hospital size. These traits may be related to perceptions of quality. Our ownership categories include: public, for-profit, and private not-for-profit (omitted). We include two measures of teaching status, hospital membership in the Council of Teaching Hospitals and the ratio of residents and interns to beds. Hospital size is measured by the number of hospital beds.

The influence of hospital traits on patient flows may depend on insurance type, reflecting either insurer influence over patient flows or systematic enrollment into certain types of coverage. For this reason we interact all of the preceding explanatory variables with each year of data and with each of the six payer types: Medicare, Medicaid, HMO, FFS, other public and other private payers. The FFS group corresponds to Commercial and BCBS insurers. ${ }^{11}$ Due to unreliability of coding for patients over the age of 65 and differences between Medicare and non-Medicare HMOs, all patients over the age of 65 were coded as Medicare patients even though some were enrolled in HMOs. ${ }^{12}$ In 1994, approximately $20 \%$ of Medicare patients were enrolled in 'at risk' HMOs in California, though we cannot

\footnotetext{
10 The issue of Kaiser hospitals is a subset of the larger issue of system affiliation. For other hospitals we do not observe system affiliation. A complete model would adjust for cannibalization of volume between system partners. Because the results seem insensitive to how the largest system is treated, we are less concerned about whether the results would be sensitive to more fully modeling other systems.

11 At a few hospitals in specific years, patients were missing payer type. This represented a very small percentage of our sample (about 100 observations out of 300,000). For these patients, we imputed a payer type of Medicare if the patient was aged 65 or more, and a payer type of HMO otherwise, because HMO was the most common at these hospitals.

12 The Office of Statewide Health Planning and Development (OSHPD) permits only one designated principal source of payment. Hospitals were instructed to code the principal source of payment for Medicare recipients enrolled in HMOs as 'Medicare' as opposed to 'HMO'. Because not all hospitals complied, there is non-random coding of the over 65 population which may introduce systematic bias. Moreover, Medicare HMOs differ from other HMOs for several reasons. First, during our study period a substantial number of Medicare HMO enrollees were in 'cost' HMOs. These HMOs are not at risk for enrollee expenditures. Expenditures are passed through to Medicare. Second, Medicare HMOs were very new during this period and their contracting may have differed from other plans. Finally, Medicare beneficiaries can disenroll from their HMO monthly, unlike other HMO enrollees. This changes the nature of the plan and may affect contracting between plans and providers. Thus elderly HMO beneficiaries may be enrolled in fundamentally different types of plans. Because we coded all elderly as Medicare beneficiaries, our coefficients should be interpreted as reflecting the blend of HMO enrollment in Medicare.
} 
Table 1

CABG entry by year

\begin{tabular}{lcccc}
\hline Year & Incumbents & Potential entrants & Entrants & Potential entrants that enter $(\%)$ \\
\hline 1985 & 84 & 142 & 1 & 1 \\
1986 & 85 & 143 & 8 & 6 \\
1987 & 93 & 136 & 10 & 7 \\
1988 & 103 & 125 & 7 & 6 \\
1989 & 110 & 120 & 7 & 6 \\
1990 & 116 & 114 & 2 & 2 \\
1991 & 117 & 111 & 4 & 4 \\
1992 & 119 & 108 & 2 & 2 \\
1993 & 121 & 107 & 4 & 4 \\
1994 & 125 & 102 & 1 & 1 \\
Total & 1073 & 1208 & 46 & 4 \\
Unique hospitals & 129 & 154 & 46 & 30 \\
\hline
\end{tabular}

Note: between 1989 and 1991 there were a total of four exits, which is why the number of incumbents between 1990 and 1992 does not match the number of entrants plus incumbents in the previous year.

reliably identify them. ${ }^{13}$ This figure was substantially smaller during most of our study period. The other private payer type includes self-pay and indigent patients, which is where uninsured patients would be classified.

The $X$ 's also include non-cardiac volume and lagged volume of cardiac services. We separately measure 1- and 2-year lags for three types of cardiac services: cardiac catheterizations, PTCA, and CABG. The lagged volumes are measured by payer type. For non-cardiac and 1-year lagged CABG volume, we included the volumes for all payer types. For the other volume variables, we included the volume only for the own payer type. Additionally, we include the patient's race interacted with the percent of non-cardiac patients of that race discharged by the hospital. The purpose of these variables is to capture unobserved traits, such as strength of referral network, related to the demand for the hospital's cardiac services. They allow us to more accurately predict quantities within the context of the reduced-form choice model. We are not as interested in testing hypotheses related to the preceding variables. For this reason the multicollinearity of many of the explanatory variables is not of concern.

Because potential entrants will have no lagged $\mathrm{CABG}$ volume, the lagged variables capture any negative association with new entrants. However, almost all entrants will have prior experience with cardiac catheterization so the lagged cardiac catheterization volumes will capture the general presence of the hospital in the market for cardiac services. Because we use lagged volumes, our entry equations only include hospitals between 1986 and 1994, even though our data set extends from 1984 to 1994.

Explanatory variables in our entry model are much fewer. The main variables of interest are the predicted volumes by payer type. The entry threshold variables (Z's) include beds, an indicator of for-profit status, COTH membership, residents per bed and year dummies.

There were 46 hospitals that entered the market for CABG between 1985 and 1994. This represents a growth of $49 \%$ over the 84 providers in 1984. Table 1 contains details on the pattern of entry and number of incumbents. On average there were 121 potential entrants in

\footnotetext{
13 Prospective Payment Assessment Commission (1995).
} 
Table 2

Descriptive statistics on hospitals

\begin{tabular}{llll}
\hline & 'In' CABG & Potential entrants & Entrants \\
\hline Beds & $342(206)$ & $180(88)$ & $247(87)$ \\
CABG distance traveled (km) & $18.50(12.2)$ & - & $14.02(9.7)$ \\
For-profit (\%) & 13 & 27 & 20 \\
Private not-for-profit (\%) & 84 & 64 & 78 \\
Public $(\%)$ & 3 & 9 & 2 \\
COTH member $(\%)$ & 15 & 3 & 2 \\
Residents per bed & $0.07(0.2)$ & $0.03(0.1)$ & $0.01(0.0)$ \\
Non-cardiac volume per year & $15,464(9561)$ & $8157(5470)$ & $11,300(4723)$ \\
Cath volume per year & $646(405)$ & $83(118)$ & $292(140)$ \\
PTCA volume per year & $245(201)$ & - & $53(56)$ \\
CABG volume per year & & - & $43(29)$ \\
Medicare & $156(145)$ & - & $3(9)$ \\
Medicaid & $16(25)$ & - & $21(19)$ \\
FFS/commercial & $51(50)$ & - & $7(9)$ \\
HMO & $40(76)$ & - & $1(2)$ \\
Other public & $5(9)$ & - & $2(3)$ \\
Other private & $10(17)$ & - & $77(47)$ \\
Total & $278(244)$ & 1066 & 45 \\
$N \quad 1034$ & & & 45
\end{tabular}

Note: each cell indicates the mean value, with the standard deviation in parentheses.

each year, ranging from 142 in 1985 to 102 in 1994. Descriptive statistics for incumbents, potential entrants, and entrants are in Table 2. ${ }^{14}$

The number of exiters can also be inferred from Table 1 by examining the difference between the number of incumbents and entrants in one year and the number of incumbents in the subsequent year. There were four hospitals that exited CABG during our sample period. Two of these were due to hospitals that closed, leaving just two genuine exits from CABG during our sample period.

\section{Results}

\subsection{Results from patient flow model}

Table 3 summarizes the estimated coefficients for our patient flow model. Because we estimate over a thousand coefficients (one set of 25 coefficients for each year and payer type), we do not present all of the coefficients. Instead, we present the mean and range of the point estimates for each of the 25 coefficients across years and payer types.

Many of the coefficients capture the same underlying hospital attributes (e.g. membership in COTH and residents per bed; 1- and 2-year lagged CABG volume; non-cardiac volume

\footnotetext{
${ }^{14}$ Hospitals that do not provide CABG also do not provide PTCA because an emergency CABG surgery may be required following a PTCA. Hence, this table does not report CABG or PTCA volumes for potential entrants, most of whom are not actual entrants, and hence do not provide CABG.
} 
Table 3

Coefficient estimates from patient flow model

\begin{tabular}{|c|c|c|c|c|c|}
\hline Coefficient type & Mean & Min. & Max. & Positive & Negative \\
\hline Beds $\times 100$ & -0.012 & -0.608 & 0.471 & 28 & 26 \\
\hline Public & -0.866 & -3.576 & 2.044 & 15 & 39 \\
\hline For-profit & -0.352 & -1.403 & 0.208 & 7 & 47 \\
\hline COTH & 0.332 & -0.817 & 2.262 & 36 & 18 \\
\hline Residents per bed & 0.248 & -2.342 & 3.197 & 31 & 23 \\
\hline $\ln ($ Dist +1$)$ & 0.017 & -0.323 & 0.795 & 23 & 31 \\
\hline$[\ln (\text { Dist }+1)]^{2}$ & -0.331 & -0.464 & -0.250 & 0 & 54 \\
\hline Lag cath volume & 0.010 & -5.923 & 4.142 & 30 & 24 \\
\hline Lag PTCA volume & 1.466 & -3.160 & 11.17 & 38 & 16 \\
\hline 2 lag cath volume & 0.237 & -2.239 & 5.980 & 31 & 23 \\
\hline 2 lag PTCA volume & 0.110 & -6.057 & 8.986 & 23 & 31 \\
\hline 2 lag CABG volume & 0.152 & -5.843 & 6.485 & 24 & 30 \\
\hline Lag CABG vol. Medicare & 0.222 & -0.183 & 1.017 & 49 & 5 \\
\hline Lag CABG vol. Medicaid & 0.781 & -1.151 & 6.104 & 44 & 10 \\
\hline Lag CABG vol. Other public & 1.063 & -3.912 & 9.964 & 36 & 18 \\
\hline Lag CABG vol. FFS & 0.030 & -1.222 & 1.132 & 33 & 21 \\
\hline Lag CABG vol. HMO & 0.116 & -1.208 & 1.835 & 34 & 20 \\
\hline Lag CABG vol. Other private & 0.559 & -3.663 & 5.649 & 36 & 18 \\
\hline Non-heart vol. Medicare & -0.208 & -2.858 & 3.367 & 24 & 30 \\
\hline Non-heart vol. Medicaid & -0.182 & -3.086 & 0.944 & 21 & 33 \\
\hline Non-heart other public & 0.675 & -6.682 & 12.15 & 29 & 25 \\
\hline Non-heart vol. FFS & 0.118 & -1.896 & 2.147 & 30 & 24 \\
\hline Non-heart vol. HMO & 0.022 & -2.394 & 2.147 & 25 & 29 \\
\hline Non-heart vol. other private & -0.2869 & -4.476 & 4.612 & 21 & 33 \\
\hline Percent own race & 1.521 & 0.836 & 3.614 & 54 & 0 \\
\hline
\end{tabular}

Note: each row pertains to 54 estimated coefficients, corresponding to 9 years of data and six payer groups. Volume is measured in units of 100 patients.

and beds; log distance plus 1 and its square). Moreover, many of the coefficient estimates change sign across year and payer type. These factors make it difficult to visually assess the marginal impact of various hospital attributes on volume.

Nevertheless, our results generally support existing models of patient flow and our expectations. For example, the marginal impact of distance is negative at all reasonable distances for all payer types. Additionally, lagged CABG volumes are positively associated with patient flows. The impact of other variables, such as being a teaching hospital, varies by payer type and by year.

We further assess the reasonableness of the patient flow model by examining the stability of the predictions across time. Table 4 reports the correlation between actual 1994 hospital volume by payer type (total and that kept in our sample after excluding patients with data problems as noted in Section 3) and predicted 1994 volumes using coefficients generated from 1994 volume and lagged data (1989-1993). The correlation between actual and predicted 1994 volumes is 0.97 . If we use coefficients generated from 1993 data to predict 1994 volume, the correlation with actual 1994 volume drops to 0.85 . Further lags have similar correlations that remain over 0.8 . The relatively high correlations are consistent with our model, which assumes that hospitals can forecast expected demand before deciding to enter. 
Table 4

Correlation between actual 1994 volume and predicted 1994 volume

\begin{tabular}{ccccccccc}
\hline & Actual 1994 & \multirow{2}{*}{$\begin{array}{l}\text { Kept from } \\
1994^{\mathrm{a}}\end{array}$} & \multicolumn{6}{c}{ Predicted 1994 volume based on coefficients from: } \\
\cline { 4 - 8 } & & & 1994 & 1993 & 1992 & 1991 & 1990 & 1989 \\
\hline Actual 1994 & 1.00 & - & - & - & - & - & - & - \\
Kept from 1994 & 1.00 & 1.00 & - & - & - & - & - & - \\
Predicted 1994 volume based on coefficients from: & & & & & \\
1994 & 0.97 & 0.97 & 1.00 & - & - & - & - & - \\
1993 & 0.85 & 0.86 & 0.89 & 1.00 & - & - & - & - \\
1992 & 0.87 & 0.88 & 0.92 & 0.90 & 1.00 & - & - & - \\
1991 & 0.81 & 0.83 & 0.86 & 0.82 & 0.96 & 1.00 & - & - \\
1990 & 0.80 & 0.81 & 0.83 & 0.82 & 0.91 & 0.91 & 1.00 & - \\
1989 & 0.84 & 0.84 & 0.88 & 0.86 & 0.89 & 0.84 & 0.93 & 1.00 \\
\hline
\end{tabular}

Note: an observation is a hospital that is 'in' the CABG market $\times$ payer type.

${ }^{a}$ Patients were omitted if they had missing distance data, traveled further than $120 \mathrm{~km}$, or received care at hospitals with too little volume to be considered 'in' the CABG market.

We are also interested in understanding whether there is selection bias in applying our coefficient estimates to potential entrants. Since our estimates are generated for all firms that are 'in' the CABG market, we can test for selection bias by examining the fit of the model for actual entrants, who form a small subset of the firms that are 'in' the market. In Table 5, we report a regression of actual volume by payer type for each year on the corresponding

Table 5

Bias in predicted volume for actual entrants

\begin{tabular}{lc}
\hline Regressor & Estimate \\
\hline Constant & $2.048^{* * *}(0.276)$ \\
Predicted volume & $0.960^{* * *}(0.003)$ \\
Predicted volume $\times$ Medicare $\times$ entry at $t$ & $-0.394^{* * *}(0.042)$ \\
Predicted volume $\times$ Medicaid $\times$ entry at $t$ & $-0.543(0.658)$ \\
Predicted volume $\times$ other public $\times$ entry at $t$ & $-1.388(1.381)$ \\
Predicted volume $\times$ FFS $\times$ entry at $t$ & $-0.261^{*}(0.097)$ \\
Predicted volume $\times$ HMO $\times$ entry at $t$ & $-0.546^{*}(0.209)$ \\
Predicted volume $\times$ other private $\times$ entry at $t$ & $-0.582(0.031)$ \\
Predicted volume $\times$ Medicare $\times$ entry at $t-1$ & $-0.059^{*}(0.031)$ \\
Predicted volume $\times$ Medicaid $\times$ entry at $t-1$ & $0.044(0.426)$ \\
Predicted volume $\times$ other public $\times$ entry at $t-1$ & $-0.517(1.086)$ \\
Predicted volume $\times$ FFS $\times$ entry at $t-1$ & $-0.051(0.068)$ \\
Predicted volume $\times$ HMO $\times$ entry at $t-1$ & $-0.204(0.154)$ \\
Predicted volume $\times$ other private $\times$ entry at $t-1$ & $-0.303(0.519)$ \\
$R^{2}$ & 0.95 \\
\hline
\end{tabular}

Note: the dependent variable is the actual kept volume. An observation is a hospital that is 'in' the CABG market $\times$ payer type $\times$ year. S.E. are given in parentheses.

$$
\begin{aligned}
& * P<0.1 \text {. } \\
& { }^{* * *} P<0.01 .
\end{aligned}
$$


Table 6

Coefficient estimates from entry model

\begin{tabular}{lccc}
\hline Variable & Base model: i.i.d. probit & Serially correlated probit & Cox model \\
\hline Medicare volume & $0.636(0.421)$ & $0.925^{*}(0.445)$ & $0.932(0.643)$ \\
Medicaid volume & $-7.550^{* * *}(3.293)$ & $-6.560(4.475)$ & $-14.26^{* *}(5.949)$ \\
Other public volume & $13.65^{*}(7.031)$ & $0.090(0.106)$ & $24.11^{*}(13.66)$ \\
FFS volume & $2.096^{* *}(1.045)$ & $2.458^{* *}(1.169)$ & $3.992^{* *}(1.640)$ \\
HMO volume & $-1.000(0.767)$ & $-0.685(0.989)$ & $-1.519(1.496)$ \\
Other private volume & $-0.899(2.029)$ & $-0.270(0.651)$ & $-1.515(3.525)$ \\
Hospital beds & $0.006^{* * *}(0.001)$ & $0.007^{* * *}(0.002)$ & $0.011^{* * *}(0.002)$ \\
COTH membership & $3.825(2.393)$ & $3.485(3.334)$ & $9.045(5.256)$ \\
For-profit & $-0.054(0.208)$ & $0.004(0.320)$ & $-0.024(0.415)$ \\
Residents per bed & $-15.33^{*}(8.881)$ & $-12.26(12.54)$ & $34.66^{*}(19.71)$ \\
Serial correlation parameter $(\rho)$ & - & $0.834^{* * *}(0.232)$ & - \\
d(Pr entry)/d(Medicare vol.) & $5.72 \%$ & $8.33 \%$ & $3.93 \%$ \\
d(Pr entry)/d(Medicaid vol.) & $-68.0 \%$ & $-59.0 \%$ & $-60.2 \%$ \\
d(Pr entry)/d(FFS volume) & $18.9 \%$ & $22.1 \%$ & $16.9 \%$ \\
d (Pr Entry)/d (HMO volume) & $-8.09 \%$ & $-6.17 \%$ & $-6.41 \%$ \\
Year dummies included? & Yes & Yes & No \\
\hline
\end{tabular}

Note: S.E. are given in parentheses. Volume is measured in units of 100 patients. Probability derivatives are calculated using the sample mean entry probability of $4.22 \%$, and for the Cox model, using the estimated mean baseline hazard of $0.186 \%$.

$$
\begin{aligned}
& * P<0.1 \text {. } \\
& \text { ** } P<0.05 \text {. } \\
& \text { *** } P<0.01 \text {. }
\end{aligned}
$$

predicted volume. ${ }^{15}$ To test for systematic bias in predicted volume for entrants relative to incumbents, we interact predicted volume with indicators for year of entry and year following entry. Entrants have significantly less volume than predicted in the year of entry. For example, in the year that a hospital enters, its actual Medicare volume is 39 percentage points lower than predicted for incumbents. The corresponding number for Medicaid is 54 percentage points. The likely reason is that they enter in the middle of the year and there may be a startup period with lower volume. In the year after they enter, their actual volumes are similar to predicted incumbent volumes, and an $F$-test does not reveal any significant differences $(P=0.38)$. While there does not appear to be selection bias for actual entrants, we have no direct way of verifying the absence of selection bias for potential entrants who did not enter.

\subsection{Results from entry model}

In Table 6, we present estimates of our entry model, using three specifications: the base model from (5) which is an i.i.d. probit, a serially correlated probit based on (6) and the Cox specification from (7). The relative magnitudes of the coefficients are consistent across the

\footnotetext{
15 The dependent variable excludes patients omitted from the sample due to the data problems noted in Section 3.
} 
different specifications. We find, for the major payers, the average returns over our study period are the most generous for FFS, followed by Medicare, HMO and Medicaid. Average Medicare returns over the period are positive, small, but statistically significant only in the serially correlated probit specification. FFS returns are always significantly positive and appear to be roughly three times that of Medicare. HMO returns are slightly below zero and never statistically significant. Medicaid consistently pays below average variable cost, with a return that is estimated to be roughly three times the magnitude for that of FFS, but with the opposite sign, and is significant except in the serially correlated probit specification.

The coefficient on serial correlation in the serially correlated probit, $\rho$, is found to be 0.834 and significant. However, the coefficients are similar in magnitude and significance across the two probit specifications. We are not aware of any direct way of testing the probit models against the Cox model and the magnitude of the coefficients are not directly comparable across the specifications. However, we can compare the magnitudes by examining the derivative of the probability of entry with respect to a change in volume by payer, evaluated for a hospital at the sample mean probability of entry. ${ }^{16}$ Table 6 reports that these magnitudes are very consistent across the specifications for the four key payer types. Hence, we focus on the i.i.d. probit for the subsequent results.

Our findings are consistent with those of Cutler and McClellan (1996), who find that HMO penetration is inversely related to entry at the state level. Our analysis demonstrates that the inverse relation between HMO penetration and entry is not because HMOs pay below variable costs but because they pay less than other payers.

By interacting volume with a time trend, we find evidence that returns for Medicare are decreasing over our sample period, and that the decrease is statistically significant (Table 7). The results show that in 1986, the start of our sample period, Medicare reimbursed with a similar return to the FFS return from Table $6(\beta=2.056$ versus 2.096), while by 1994 , returns for Medicare are negative, $(\beta=-0.832) .{ }^{17}$ The small Medicare coefficient in Table 6 reflects the average of high early period returns and low or negative later period returns. The drop in returns for Medicare is consistent with published Medicare returns, which show a similar decrease in overall hospital reimbursement rates over our sample period. ${ }^{18}$

In contrast to the Medicare results, we do not find any other statistically significant time variation in returns. Medicaid returns are shown to be increasing during our sample period, though the increase is not statistically significant at the 0.10 level.

The estimates presented above assume that average returns are invariant to market competition. We explore this hypothesis by interacting the predicted volume estimates for each

\footnotetext{
${ }^{16}$ For the Cox specification, this should be interpreted as the derivative of the cumulative probability of entry over a 1 year period conditional on no entry at the start of the period.

17 Recall all patients over 65 were recoded as Medicare patients even though some were enrolled in HMOs. This recoding addressed data problems and conceptual issues associated with Medicare HMOs. Without the recoding the declining trend in Medicare returns, which we know from secondary sources existed, disappears. Thus, we trust the results with the recoded data.

18 Prospective Payment Assessment Commission (1995).
} 
Table 7

Changes in returns over time

\begin{tabular}{lc}
\hline Variable & Estimate \\
\hline Medicare volume & $2.056^{* *}(0.827)$ \\
Medicaid volume & $-14.64^{* *}(6.755)$ \\
Other public volume & $17.94(13.21)$ \\
FFS volume & $0.565(1.490)$ \\
HMO volume & $-1.585(1.566)$ \\
Other private volume & $-3.189(3.266)$ \\
Medicare volume $\times$ year & $-0.361^{* *}(0.184)$ \\
Medicaid volume $\times$ year & $1.600(1.371)$ \\
Other public volume $\times$ year & $-1.625(3.100)$ \\
FFS volume $\times$ year & $0.239(0.540)$ \\
HMO volume $\times$ year & $0.187(0.357)$ \\
Other private volume $\times$ year & $2.358(1.879)$ \\
Hospital beds & $0.006^{* * *}(0.001)$ \\
COTH membership & $4.248^{*}(2.500)$ \\
For-profit & $-0.069(0.211)$ \\
Residents per bed & $-18.60^{* *}(9.409)$ \\
\hline
\end{tabular}

Note: S.E. are given in parentheses. Volume is measured in units of 100 patients. Time is measured from a base year of 1986, so that 1986 is Year 0 and 1994 is Year 8. Year dummies are included in specification.

$$
\begin{aligned}
& * P<0.1 . \\
& { }^{* *} P<0.05 \\
& { }^{* * *} P<0.01
\end{aligned}
$$

hospital with a Herfindahl for that hospital. ${ }^{19}$ The Herfindahl is based on share of CABG admissions for hospitals within a $30 \mathrm{~km}$ radius of the hospital in question. ${ }^{20}$ Separate Herfindahl indices are created each year for each hospital and are not payer specific.

The results suggest that HMO returns increase with decreased competition (or equivalently, with increased Herfindahl) (Table 8). None of the other interactions with competition are statistically significant. HMO returns approximate FFS returns from Table 6 in markets with a Herfindahl of 0.616 . HMO returns approximate 0 in markets with a Herfindahl equivalent to 0.398 (approximately 2.5 equal sized hospitals). This is close to the mean Herfindahl in our sample (0.395). Bresnahan and Reiss (1991) also report that most of the gains to competition are achieved with relatively few providers, and our estimates suggest competition in the market for HMO patients is even more vigorous. Given the amount of search in the HMO market, this is not surprising. In contrast, the statistically insignificant coefficients on the FFS and Medicare interactions suggest that these returns are not much affected by competition.

\footnotetext{
$\overline{19}$ Returns would also be affected by competition in the insurance market (including among HMOs). We do not measure this competition.

${ }^{20}$ Competition may influence returns for Medicare and Medicaid patients if there is quality competition, which increases costs.
} 
Table 8

Impact of competition on returns

\begin{tabular}{lc}
\hline Variable & Estimate \\
\hline Medicare volume & $1.358(0.839)$ \\
Medicaid volume & $-2.414(7.102)$ \\
Other public volume & $28.74^{* *}(14.04)$ \\
FFS volume & $1.421(1.499)$ \\
HMO volume & $-3.833^{*}(2.061)$ \\
Other private volume & $-6.501(4.127)$ \\
Medicare volume $\times$ Herfindahl & $-1.690(1.321)$ \\
Medicaid volume $\times$ Herfindahl & $-6.822(9.060)$ \\
Other public volume $\times$ Herfindahl & $-29.79(21.60)$ \\
FFS volume $\times$ Herfindahl & $0.890(2.872)$ \\
HMO volume $\times$ Herfindahl & $9.629^{*}(5.428)$ \\
Other private volume $\times$ Herfindahl & $27.57(12.75)$ \\
Hospital beds & $0.007^{* *}(0.001)$ \\
COTH membership & $4.753^{*}(2.512)$ \\
For-profit & $0.006(0.224)$ \\
Residents per bed & $-17.99^{*}(9.358)$ \\
\hline Not S.E. are given in parentheses. Volume is measured & units 0 (100)
\end{tabular}

Note: S.E. are given in parentheses. Volume is measured in units of 100 patients. Year dummies are included in specification.

$$
\begin{aligned}
& { }^{*} P<0.1 . \\
& { }^{* *} P<0.05 . \\
& { }^{* * *} P<0.01 .
\end{aligned}
$$

\subsection{Sensitivity analysis}

We examine the sensitivity of our results to three possible effects: unobserved market traits that are correlated with both entry and payer mix, demand expansion from entry, and the endogeneity of volume.

First, note that the results presented above are partly identified on the basis of the payer mix of the population in the geographic area surrounding the potential entrants. If unobserved market characteristics are correlated with payer mix, the results may bias our estimates of returns. For example, assume that hospitals in areas with a large number of Medicaid recipients have a difficult time attracting cardiac surgeons or that high Medicaid volume is correlated with a high percentage of uninsured. ${ }^{21}$ We will predict a high Medicaid volume for potential entrants in these areas and a low probability of entry. This will lead to an inference of low or negative margins. To test for such market specific effects, we re-estimate the entry model with additional explanatory variables that measure the mean payer mix for all admissions in the hospital's market. ${ }^{22}$ For this specification, the identification of the returns will come from the level of competition for different types of patients.

\footnotetext{
${ }^{21}$ Uninsured patients are included in the 'other private' category, but if the proportion of uninsured in that category varies with Medicaid enrollment there may still be a measurement issue.

22 We define the market to be the set of people who live within $120 \mathrm{~km}$ of the hospital who were discharged from a hospital in California.
} 
Table 9

Sensitivity analysis

\begin{tabular}{lccc}
\hline Variable & Including payer mix & Including CABG rate & Instrumental variables \\
\hline Medicare volume & $0.493(0.452)$ & $0.335(0.455)$ & $0.046(0.037)$ \\
Medicaid volume & $-4.920(4.061)$ & $-7.580^{*}(0.413)$ & $-0.541^{* *}(0.192)$ \\
Other public volume & $6.599(7.898)$ & $9.156(8.279)$ & $0.734(0.555)$ \\
FFS volume & $3.704^{* * *}(1.182)$ & $3.606^{* * *}(1.173)$ & $0.309^{* * *}(0.093)$ \\
HMO volume & $-0.879(0.810)$ & $-0.553(0.758)$ & $-0.059^{* *}(0.024)$ \\
Other private volume & $-0.490(2.282)$ & $-0.969(2.456)$ & $-0.140(0.145)$ \\
Medicare market share & $10.58^{*}(5.888)$ & - & - \\
Other public market share & $41.99^{* * *}(13.49)$ & - & - \\
FFS market share & $5.718(5.847)$ & - & - \\
HMO market share & $10.49^{* *}(4.739)$ & - & - \\
Other private market share & $8.114(13.78)$ & - & - \\
Medicare CABG rate & - & $3.512(14.08)$ & - \\
Medicaid CABG rate & - & $18.16^{* *}(8.042)$ & - \\
Other public CABG rate & - & $5.977^{* * *}(2.257)$ & - \\
FFS CABG rate & - & $-15.73(10.23)$ & - \\
HMO CABG rate & - & $6.547(4.522)$ & - \\
Other private CABG rate & - & $6.689(5.131)$ & \\
\hline
\end{tabular}

Note: S.E are given in parentheses. Volume is measured in units of 100 patients. Year dummies, beds, COTH, for-profit and residents per bed are included in all specifications.

$$
\begin{aligned}
& * P<0.1 \\
& { }^{* *} P<0.05 \\
& * * * P<0.01
\end{aligned}
$$

These estimates yield very similar predictions to the base model, except that the Medicaid market share is no longer significant, and the absolute value of the point estimate on Medicaid returns drops by about 35\% (Table 6 column 1 versus Table 9 column 1). Since Medicaid market share is the omitted category, the positive signs on all the other coefficients suggest that areas with high Medicaid penetration are relatively unattractive. In contrast, the estimates suggest that areas with high HMO penetration and high Medicare penetration are attractive for entrants, even controlling for predicted volume. Perhaps it is easy to attract physicians to these areas, lowering the fixed costs of entry.

A second issue related to our base results is that we have not allowed for the possibility that new entrants may expand the demand for CABG surgery. Specifically, evidence suggests that aggregate volume will rise with entry simply because of reduced distance from patients to CABG providers (Cutler and McClellan, 1996). To explore the sensitivity of our results to potential demand expansion, we include estimates of the percentage of ischemic heart disease admissions in each hospital's market area that received CABG. ${ }^{23}$ A higher percentage would suggest a lower potential for demand expansion and thus a lower probability of entry. Therefore, a negative sign on the CABG rate is consistent with the ability for significant demand expansion for that payer.

The results display the same pattern of results as the base analysis, although some of the coefficients change in magnitude. Most notably, the estimated FFS returns rise by about

\footnotetext{
23 Following Langa and Sussman (1993), we use the following ICD-9 diagnosis codes for ischemic heart disease: 410.xx, 411.1, 411.8, 413.xx, 414.0x, 414.8x, and 414.9x.
} 
$70 \%$ in this specification (Table 6 column 1 versus Table 9 column 2), which strengthens our earlier finding that FFS payers generate a positive return. This is supported by the large and negative, though statistically insignificant, coefficient on the FFS CABG rate which suggests that entry is more likely when inducement opportunities are high (low CABG rate). In contrast, the large, positive and statistically significant coefficient on the Medicaid $\mathrm{CABG}$ rates suggests that entrants are more likely if there is little room to expand $\mathrm{CABG}$ surgery for Medicaid patients. This supports the earlier finding that Medicaid does not provide sufficient returns because it is consistent with the hypothesis that hospitals do not want to enter areas where they may be forced to provide services to patients whose insurers do not provide sufficient returns.

Finally, the predicted volume that we use in our entry model is potentially endogenous because it is based on our assumption of rational expectations regarding competitive interactions. Specifically, in our base analysis, we calculate predicted volume for any potential entrant using as the choice set all hospitals that actually provide $\mathrm{CABG}$ services in the year of entry. Thus, the predicted volume for any potential entrant reflects the unobserved factors driving competitors to enter. Because we assume the potential entrant observes these factors, there is a correlation between entrants' predicted volumes and the unobserved component of their entry decisions. ${ }^{24}$

To explore the sensitivity of our results to this endogeneity we estimate a linear probability model of entry using IV techniques. Our instruments consist of predicted volume calculated assuming only the potential entrant and incumbents were in the market. Any unobserved factors that influence competitors to enter are not reflected in this instrument, eliminating the cause of the endogeneity. The instruments are essentially functions of the characteristics of the competing firms, aggregated in a manner that emphasizes the proximity of the competitors to the potential entrant in product space. ${ }^{25}$

We find that the use of instruments yields conclusions similar to those derived from our primary analysis (Table 9, column 3). ${ }^{26}$ While it is not possible to directly compare the IV coefficients to the coefficients from our base model which assumed the hazard function was based on a normal distribution of the entry equation error, the signs and significance of all the coefficients of interest remain the same. Moreover, a Hausman test of the IV model against a linear probability model fails to reject the consistency of the non-IV model $(P=0.50)$.

\section{Implications and conclusions}

Estimating the returns by payer type that hospitals receive from the provision of high technology medical services is important. There has been substantial concern among policy makers regarding 'over diffusion' of expensive medical technologies such as CABG,

\footnotetext{
${ }^{24}$ Berry (1992) focuses on this issue in an examination of entry among airlines.

25 Similarly, Frank and Salkever (1991) use characteristics of competing hospitals to instrument for endogenous selection into insurance, in a study of the provision of charity care.

26 The similar results are in spite of the fact that a Hausman test suggests the presence of endogeneity in our volume coefficients.
} 
which could be driven by excess returns to the provision of such services. Yet, accounting complexities and case-mix variation make direct assessment of returns difficult. We rely on entry decisions, together with a structural model of entry, to infer returns. The analysis not only confirms widely held opinions about the relative generosity of payments, but also presents new evidence regarding the magnitude of returns, and the changes in these returns over time and across different levels of competition.

Our analysis reveals that Medicare returns for CABG have followed the same pattern of aggregate Medicare returns: generous in the mid-1980s, but approximating zero by the early/mid-1990s. Only Medicare returns were sensitive to time. Throughout our study period, HMOs appear to reimburse hospitals at approximately average variable costs. This result is consistent with models of strong price sensitive search by HMOs and the absence of hospital market power in this payer segment. Although our results suggest that HMOs are not contributing to fixed costs, they are not paying substantially below variable costs as some of their opponents contend. In contrast, Medicaid payments seem below variable costs. Commercial and BCBS payers pay the most generous returns, which appear to have been relatively constant over time. By the end of the study period, only these payers are contributing to fixed costs. Finally, our results suggest that HMO returns (and only HMO returns) are particularly sensitive to competition among CABG providers. We find that it takes between two and three CABG providers in the market to drive returns from HMOs to zero.

Most experts predict continued growth in enrollment in HMOs and in other plans which shop for hospitals based on price. Our results indicate that this will mean fewer payers that contribute to fixed costs. Thus, future entry into CABG is likely to be much slower and exit from the market is possible. Mergers by hospitals to gain market power in their dealings with HMOs are also likely. In this scenario, returns from HMOs and other strongly managed plans are likely to grow. This implies that cost savings achieved by HMOs through efficient purchasing may diminish.

Moreover, to the extent that our results are generalizable to other services, they suggest that there will be a slowdown in the rate of entry for other high technology services in the hospital sector. Although we cannot assess the health consequences of use, which is necessary to quantify whether there is 'over-diffusion', our results suggest that hospitals continue to have the incentive to encourage CABG procedures for FFS patients at the margin. In contrast, HMOs do not give hospitals this incentive and by the end of our study period, Medicare payment rates have also removed the hospital incentive to provide this surgery. The lower payment by Medicaid suggests an incentive for lower utilization, implying that these patients will likely face continued access difficulties. ${ }^{27}$

While our estimation appears to give reasonable results, there are several modeling and econometric specification issues that arise. First, we make the restrictive assumption that, holding the Herfindahl constant, average returns are fixed for a given payer type. Second, we have treated our predicted volumes as not having any measurement error, even though this may occur for a finite sample of patients. Third, we have not incorporated dynamics into our return function.

\footnotetext{
$\overline{27}$ Langa and Sussman (1993) report that during the mid-1980s, rates of revascularization (CABG and PTCA) grew the least in the Medicaid population.
} 
We feel that this work makes two methodological contributions to the general health economics and industrial organization literature. First, this paper shows how to construct an entry model where markets are endogenously determined. Specifically, our method uses a patient flow model to base market definitions on an estimated demand structure. In contrast, existing entry papers have used pre-determined geographic market definitions. This is potentially problematic because these pre-determined markets are relatively inflexible in their ability to control for differing degrees of product differentiation created largely by differential distances between competitors within the market.

Second, we feel that this paper lends credence to the general modeling approach of assuming positive returns from entry, and using this to infer structural parameters. Other authors have either focused on different motives for hospital entry into high technology services (e.g. the medical arms race; see Luft et al. (1986) and Dranove et al. (1992)) or have been more agnostic regarding the motives for entry (e.g. Cutler and McClellan (1996)). We believe that our emphasis on returns is justified because our results are both consistent with economic theories and with accounting data. Other models would have to explain features of the data such as why only Medicare returns decline significantly over time and why only HMO returns respond significantly to competition. Thus, our results suggest that it is a valid and useful endeavor to estimate entry models for other industries in order to infer fundamental parameters that are of economic interest.

\section{Acknowledgements}

We thank Rajesh Bandekar for able research assistance and the Data User Support Group of the State of California Office of Statewide Health Planning and Development for provision of the data. We acknowledge helpful comments received from Dan Ackerberg, Lanier Benkard, Steve Berry, Jim Burgess, David Cutler, Randy Ellis, Joseph Newhouse, Ariel Pakes, John Rust, seminar participants from several institutions and two anonymous referees. The views expressed herein are solely those of the authors, and do not represent those of the Federal Reserve Bank of San Francisco or of the Federal Reserve System.

\section{References}

Allison, P.D., 1995. Survival Analysis Using the SAS System: A Practical Guide. SAS Institute, Cary, NC.

Baker, L.C., Wheeler, S.K., 1998. Managed care and technology diffusion: the case of MRI. Health Affairs 17 (5), 195-207.

Berry, S.T., 1992. Estimation of a model of entry in the airline industry. Econometrica 60 (4), 889-917.

Berry, S.T., 1994. Estimating discrete-choice models of product differentiation. RAND Journal of Economics 25 , 242-262.

Bresnahan, T., Reiss, P., 1991. Entry and competition in concentrated markets. Journal of Political Economy 99 (5), 977-1009.

Burns, L.R., Wholey, D.R., 1992. The impact of physician characteristics in conditional choice models for hospital. Journal of Health Economics 11 (1), 43-62.

Chernew, M., Scanlon, D., Hayward, R., 1998. Insurance type and choice of hospital for open heart surgery. Health Services Research 33 (3), 447-466.

Cutler, D.M., McClellan, M., 1996. The Determinants of Technological Change in Heart Attack Treatment. NBER Working Paper Series. Working Paper 5751. 
Dranove, D., Shanley, M., Simon, C., 1992. Is hospital competition wasteful? RAND Journal of Economics 23 (2), 247-262.

D’Agostino, R.B., Lee, M.L., Belanger, A.J., Cupples, L.A., Anderson, K., Kannel, W.B., 1990. Relation of pooled logistic regression to time dependent Cox regression analysis: the Framingham heart study. Statistics in Medicine 9 (12), 1501-1515.

Frank, R., Salkever, D.S., 1991. The supply of charity services by non-profit hospitals: motives and market structure. RAND Journal of Economics 22 (3), 430-444.

Garnick, D.W., Lichtenberg, E., Phibbs, C.S., Luft, H.S., Peltzman, D.J., McPhee, S.J., 1989. The sensitivity of conditional choice models for hospital care to estimation technique. Journal of Health Economics 8, 377-397.

Geweke, J., 1989. Bayesian inference in econometric models using Monte Carlo integration. Econometrica 57, 1317-1339.

Hadley, J., Feder, J., 1985. Hospital cost shifting and care for the uninsured. Health Affairs 4 (3), 67-80.

Hajivassiliou, V.A., McFadden, D., Ruud, P., 1996. Simulation of multivariate normal rectangle probabilities and their derivatives: theoretical and computational results. Journal of Econometrics 72, 85-134.

Keane, M., 1994. A computationally practical simulation estimator for panel data. Econometrica 62, 95-116.

Langa, K.M., Sussman, E.J., 1993. The effect of cost-containment policies on rates of coronary revascularization in California. New England Journal of Medicine 329 (24), 1784-1789.

Luft, H.S., Robinson, J.C., Garnick, D.W., Maerki, S.C., McPhee, S.J., 1986. The role of specialized clinical services in competition among hospitals. Inquiry 23, 83-94.

Luft, H.S., Garnick, D.W., Mark, D.H., Peltzman, D.J., Phibbs, C.S., Lichtenberg, E., McPhee, S.J., 1990. Does quality influence choice of hospital? Journal of The American Medical Association 263 (21), 2899-2906.

McFadden, D., 1973. Conditional Logit Analysis of Qualitative Choice Behavior. In: Zarembka, P. (Ed.), Frontiers in Econometrics. Academic Press, New York.

McFadden, D., 1974. The measurement of urban travel demand. Journal of Public Economics 3, 303-328.

Morrisey, M., 1995. Movies and myths: hospital cost shifting. Business Economics 30, 22-25.

National Center for Health Statistics, 1986. National Hospital Discharge Survey. Detailed Diagnoses and Procedures for Patients Discharged from Short Stay Hospitals, United States, 1984. Vital Health Statistics, Vol. 13, no. 86 .

National Center for Health Statistics, 1997. National Hospital Discharge Survey. Detailed Diagnoses and Procedures. 1994. Vital Health Statistics, Vol. 13, no. 127.

Phibbs, C.S., Mark, D.H., Luft, H.S., Peltzman-Rennie, D.J., Garnick, D.W., Lichtenberg, E., McPhee, S.J., 1993. Choice of hospital for delivery: a comparison of high-risk and low-risk women. Health Services Research 28 (2), 201-222.

Prospective Payment Assessment Commission, 1995. Medicare and the American Health Care System Report to Congress.

Spetz, J., Baker, L.C., 1999. Has Managed Care Affected the Availability of Medical Technology? Public Policy Institute of California, San Francisco, CA. 